\title{
Developing an integrated longitudinal antimicrobial curriculum in an allopathic medical school
}

\author{
Gagani Athauda', Rebecca L Toonkel², Sanaz B Kashan² \\ ${ }^{I}$ Cellular Biology and Pharmacology, Herbert Wertheim College of Medicine, Florida International University, USA, \\ ${ }^{2}$ Department of Humanities, Health, and Society, Herbert Wertheim College of Medicine, Florida International \\ University, USA
}

Background: Students entering the third year clinical clerkship rotations at HWCOM have expressed a lack of confidence in their antimicrobial pharmacology knowledge. We aim to remedy this and strengthen the knowledge of the antimicrobial pharmacology as well as infectious diseases across all four years of the allopathic medical curriculum.

Methods: We are developing an innovative curriculum with multiple pedagogies which consist of flipped classroom sessions, large group case based discussions, patient note write ups and small group mini case discussions. Cases integrate microbiology, physiology, pathophysiology, evidence based medicine (EBM), pharmacology, clinical skills and infectious diseases. A visual tool has been developed to aid the study of antibiotics' spectrum of activity. Students' performance related to the intervention is assessed using in-house and/or NBME MCQ questions and a grading rubric developed by the faculty.

Results: Outcome measures of the intervention are assessed at P2-P3 interface (pre-clinical students about to enter clinical clerkships) and P4-residency interface (students about to graduate medical school). Assessments consist of USMLE style MCQ's to assess antimicrobial pharmacology knowledge to treat infectious diseases and a short survey using a 5-point Likert scale is administered to assess their level of confidence in the subject matter. Each MCQ is mapped to a specific learning objective/s, and will be used to identify gaps in knowledge. The antimicrobial curriculum will be modified to fill the identified gaps.

Conclusions: developing an integrated longitudinal antimicrobial curriculum in an allopathic medical school will equip the pre-clinical students entering the clinical clerkships and 4th year medical students entering residency training to build their confidence and knowledge of antimicrobials in treating infectious diseases and strengthen the knowledge of both the antimicrobial pharmacology as well as infectious diseases. 